\section{Quantum effects in narrow channels}

Sir-John Maddox's recent piece' "But how thin is a thin wire?". takes into account the size of the quantum effects on an electrical current conducted by a wire so thin that the current is affected by those quantum effects. A simple analysis of superfluids in narrow channels of a solid superleak. "Controversial drops" was also published" some months ago. In this article an experiment is suggested to analyse the nature of the outcoming drops from a solid superleak after the transit of the superfluid ${ }^{+} \mathrm{He}$ through the superleak.

Taking into account the quantum nature of the superfluid and that the size of a narrow channel of a solid superleak, in practice, can be some orders of magnitude smaller than a thin wire, I wonder if the two cases are similiar, if not identical. In both cases the same question arises: how narrow is the narrow channel of a solid superleak?

An interesting situation would be the case of a solid magnetic superleak ${ }^{3}$ through which the magnetic phases of superfluid $\mathrm{He}$ were in transit. In particular, A. Leggett (personal communication, 1977) said, ". . . if in these superleaks the adequate magnetic fields could be produced, quite a new kind of experiment in superfluid ${ }^{3} \mathrm{He}$ could be made, even its polarization". But, to my knowledge, no experiments with this kind of solid magnetic superleaks, with the magnetic phases of superfluid ${ }^{3} \mathrm{He}$, have yet been made.

Clavecimbellaan 273 J.M. Goldschvartz

2287 VK Rijswijk ( $\mathrm{ZH}$ ),

The Netherlands

1. Maddox. J. Nature 328, 197 (1987)

2. Goldschvartz, J M Cryogenics 26, 692-693 (1986)

3. Goldschvartz. J.M. Nature 266, 824-825 (1977).

\section{Lightning triggering and synchronization}

SIR-It has long been known that lightning causes magnetospheric 'whistlers', and the related precipitation of highenergy trapped electrons into the ionosphere has been demonstrated. Recently, Armstrong' has presented evidence suggesting that magnetospheric phenomena may influence the lightning which causes whistlers, at least in the sense that these phenomena may sometimes be synchronized with inter-hemispheric whistler echoes. Armstrong also reviews previous suggestions of how lightning may be triggered by external events. Both Armstrong ${ }^{1}$ and Rycroft $^{2}$, in an accompanying introductory article, suggest rather complex triggering mechanisms whose difficulties are recognized by the authors.

A simpler mechanism for the triggered synchronization of ordinary lightning by

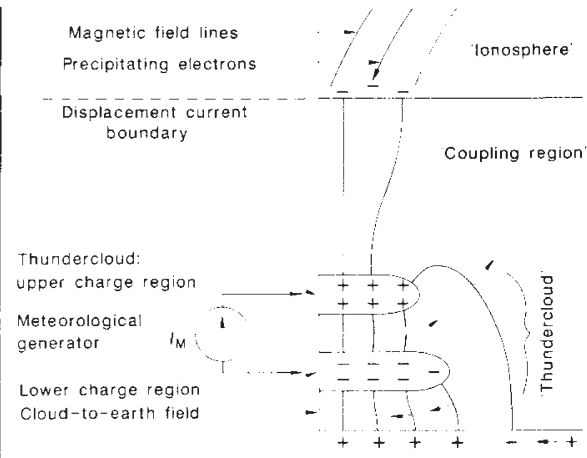

Capacitive coupling of charge from ionosphere to thundercloud. Negative electrons deposited on upper 'plate' cause field reconfiguration (indicated by transverse arrows) with more flux lines connecting from cloud-to-ionosphere, hence fewer lines from lower to upper charge but more to Earth, creating a larger cloud-toEarth field

precipitated electrons is suggested here, based on 'capacitive' coupling, in which displacement current couples charge variations from the ionosphere to the thundercloud. A simplified schematic of the fields and processes associated with a thundercloud is shown in the figure. A 'meteorological' charge separation process $\left(I_{\mathrm{M}}\right)$ maintains upper positive $\left(Q_{U}\right)$ and lower negative $\left(Q_{\mathrm{L}}\right)$ charges. The top-to-bottom cloud field is charged directly, and the cloud-to-Earth field is charged via more circuitous paths, including the ionosphere and global circuit ${ }^{3}$. Breakdown is usually initiated from the lower negative charge, presumably due to exceeding a critical electrical field ${ }^{4}$. Precipitated electrons produce an ionospheric charge separation which deposits a negative charge, $\Delta Q$, on the 'upper plate' of the cloud-to-ionosphere capacitance. (This displacement current boundary occurs where the 'relaxation time' is approximately equal to the timescale of the phenomena ${ }^{5}$, which occurs at night at $\sim 85 \mathrm{~km}$, where there is typically a sharp ledge in conductivity ${ }^{3}$.) This charge initiates a comparable decrease in the cloud's top-to-bottom electric flux which induces an increase in the cloud-to-Earth electric field $\approx \Delta Q / Q_{\mathrm{L}}$.

Triggering and synchronization can be accomplished using the following argument. Electric pulses of $P \%$ of the breakdown field, superimposed on a field increasing at $R \%$ per second, can synchronously trigger lightning for pulse spacings $T$ for which $P>R T$. Consider a case where $R$ is of the order of $1 \% \mathrm{~s}^{-1}$ and $Q_{\mathrm{L}}$ is taken as a nominal $100 \mathrm{C}$. Ionospheric charge separation of several coulombs capacitively coupled to the thundercloud would be required for synchronization of lightning-induced whistlers with echoes spaced by several seconds. Electric field measurements made in the ionosphere ${ }^{6}$ associated with lightning and whistlers, when combined with appropriate ionospheric conductivities and area coupling to the thundercloud, imply a charge separation of several coulombs, but the authors caution that non-ohmic processes may be involved. It is important to determine the actual ionospheric currents associated with lightning and whistler echoes to assess the suggested mechanism.

A recent computer model of transient ionospheric fields of lightning due to Baginski $i^{5}$ shows that $\sim 10 \%$ of the charge lowered to Earth in a cloud-to-Earth lightning stroke is separated in the ionosphere on a timescale of milliseconds after the stroke. (The model, which uses Maxwell's equations, also shows that quasistatic arguments, such as are used here, are valid on this timescale.) As larger lightning strokes can separate tens of coulombs ${ }^{4}$, this may be suffient to explain the observation that lightning is sometimes triggered by other lightning at distances up to tens of kilometres. It is noted that such triggering, which may also be associated with solar-activity-related, highenergy electron precipitation events, would tend to increase the proportion of cloud-to-Earth flashes. As cloud-to-Earth flashes contribute directly to the global circuit currents ${ }^{3}$, the cumulative effects of both solar-related and subsequently triggered cloud-to-Earth lightning may help explain the increases related to solar/ geomagnetic activity in the fair weather field and ionospheric potential reported by numerous workers and summarized by the late D.E. Olson?

\section{Communications and Space Sciences}

Laboratory,

The Pennsylvania State University,

University Park, Pennsylvania 16802, USA

1. Armstrong, W.C. Nature 327, 405-407 (1987)

2. Rycroft, M.J. Nature 327, 368-369 (1987)

Hale, L.C. in Weather and Climate Responses to Solar Variations (ed. McCormac, B.M.) 311-321 (Colorado Associated University Press, Boulder, Colorado, 1983)

4. Uman, M.A. in Lightning (Dover, New York, 1984)

Hale, L.C. \& Baginski, M.E. Nature 329, 814-816 (1987)

6. Kelley, M.C. et al. J. geophys. Res. 90, 9815-9823 (1985).

7. Olson, D.E. in Weather and Climate Responses to Solar Variations. (ed. McCormac, B.M.) 483-488 (Colorado Associated University Press, Boulder, Colorado 1983).

\section{Why plants are green}

SIR-Goldsworthy ${ }^{1}$ ascribes the light absorption properties of the photosynthetic pigments of higher plants to initial competition with prokaryotic organisms that contain bacteriorhodopsin. In this context it is wise to recall the limitations that the chemistry of the pigments places on the plasticity of photosynthetic systems. The green colour of plants could then be argued to be an 'accidental' choice forced on them because of the spectral limitations of those otherwise immensely adaptable components of bioenergetic membranes - the metalloporphyrins.

It would be odd to argue, for example, that the prosthetic group of haemo- 